DETERMINATION OF VACANCY MECHANISM FOR GRAIN BOUNDARY

SELF-DIFFUSION BY COMPUTER SIMULATION

R.W. Balluffi ${ }^{\dagger}$, Thomas Kwok*, P.D. Bristowe ${ }^{\dagger}$, A. Brokman ${ }^{\dagger+}$, P.S. Ho ${ }^{* *}$, and Sidney Yip*

tDepartment of Materials Science and Engineering, Massachusetts Institute of Technology, Cambridge, MA 02139.

* Department of Nuclear Engineering, Massachusetts Institute of Technology, Cambridge, MA 02139.

${ }^{* *}$ T. J. Watson Research Center, IBM Corporation, Yorktown Heights, NY 10598.

JUNE 1981

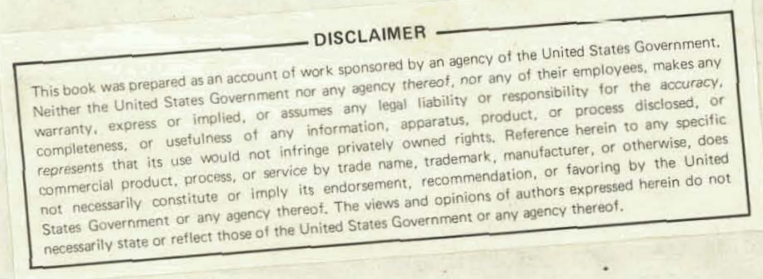

Massachusetts Institute of Technology

Cambridge, Massachusetts 02139
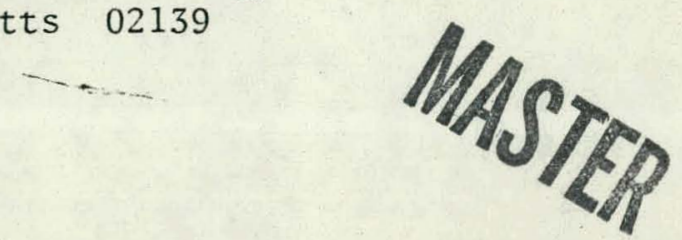

Prepared for

U.S. Department of Energy

under Contract DE-AS02-78ER05002

This report was prepared as an account of worl sponsored by the United States Golerment. Neither the United States nor the United States Department of Encray, nor any of their cuployees, nor any of their contractors, subcontractors, or their employecs, makes any waranty, express or implied, or assumes any legal liability or responsibility for the accuracy, completeness, or usefulness of any infornation, apliatus, product or process disclosed or represents that its use would not infringe privately owned rights.

+ Also at Bergman School of Applied Science, Hebrew University of Jerusalem, Israel. 


\section{DISCLAIMER}

This report was prepared as an account of work sponsored by an agency of the United States Government. Neither the United States Government nor any agency Thereof, nor any of their employees, makes any warranty, express or implied, or assumes any legal liability or responsibility for the accuracy, completeness, or usefulness of any information, apparatus, product, or process disclosed, or represents that its use would not infringe privately owned rights. Reference herein to any specific commercial product, process, or service by trade name, trademark, manufacturer, or otherwise does not necessarily constitute or imply its endorsement, recommendation, or favoring by the United States Government or any agency thereof. The views and opinions of authors expressed herein do not necessarily state or reflect those of the United States Government or any agency thereof. 


\section{DISCLAIMER}

Portions of this document may be illegible in electronic image products. Images are produced from the best available original document. 


\title{
DETERMINATION OF VACANCY MECHANISM FOR GRAIN BOUNDARY SELF-DIFFUSION BY COMPUTER SIMULATION
}

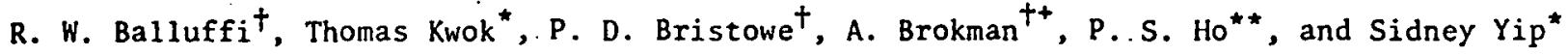 \\ + Department of Materials Science and Engineering, Massachusetts Institute of \\ Technology, Cambridge, MA 02139. \\ - Department of Nuclear Engineering, Massachusetts Institute of Technology; \\ Cambridge, MA 02139. \\ * T. J. Watson Research Center, IBM, Yorktown Heights; NY 10598.
}

\section{Introduction.}

It is currently well established that the fast self-diffusion which occurs along grain boundaries (GBs) in metals must occur by a point defect exchange mechanism. For example, it is known that rapid GB diffusion can transport a net current of atoms along GBs during both sintering (1) and diffusional creep (2), and that the two species in a binary substitutional alloy diffuse at different rates in GBs (3). However, it has not been possible to establish firmly whether the defect mechanism involves the exchange of atoms with vacancy or interstitial point defects. It has been suspected that the vacancy exchange mechanism must apply $(3,4)$, but it has been difficult to prove this hypothesis because of a lack.of detailed information at the atomistic level.

In this note we report on the results of an effort to establish the GB self-diffusion mechanism in a bcc iron $\sum=5\left(36.9^{\circ}\right)$ [001] (310) tilt boundary using the combined methods of computer molecular statics and molecular dynamics simulation. The methods are found to be complementary since the statics method efficiently provides information about the static defect energies and configurations, whereas the dynanics method allows one to observe the atomic motions associated with point defect jumps at temperatures equal to or greater than half the melting temperature. An account of the molecular dynamics results has been presented in Ref. 5 which gives. evidence for fast vacancy migration in the present GB. In the present note we review the relevant dynamics work briefly and show how the results can be combined with the results of molecular statics calculations to provide quantitative microscopic evidence in favor of the vacancy mechanism.

The simulation model used in both the static and dynamic calculations consisted of 10 layers of [001] atomic planes with their normal along the tilt axis. Each plane contained 40 atom sites, and a portion of the model containing the boundary midplane and two periods of the synmetric GB is shown in Fig. 1. All atoms were assumed to interact via a central force pairwise potential of the empirical type with a force cutoff midway between second and third neighbors (6).

\section{Molecular Statics Results}

The molecular statics technique employed a modified method of steepest descent to minimize the potential energy of the atomic ensemble (7). All entropic effects arising from lattice vibrations and kinetic energy were therefore ignored so that the simulation effectively proceeded at zero absolute temperature.

The perfect GB structure in the absence of any point defects was first determined by relaxing the initial structure in which all atoms were located on the $\sum=5$ coincidence site lattice. This resulted in a minimum energy GB structure characterized by a rigid-body translation of one

- Also at Borgman Schonl, of $\Lambda$ pplied Science, Hebrew University. of Jerusalem, Israel. 
crystal with respect to the other along [ij30] causing an expansion of $0.18 a_{0}$ (a $a_{0}=1$ attice parameter).

A vacancy was then inserted at a site in the $G B$ by removing the atom occupying that site, placing it on the surface of a reference crystal, and again relaxing the model. Configurations and energies of vacancies introduced at the sites A, B, C, and D (Fig. 1) by this procedure were determined. In each case the vacancy remained quite localized and recognizable as an empty site in the GB in agrecment with expectations based on previous work $(8,9)$. The formation energies, $E_{B}^{F}$, of these vacancies are 1 isted in Table $I$ along with their binding energies to the $G B$, $E_{B}^{B}$, determined from $E_{B}^{B}=E_{B}^{F}-E_{L}^{F}$, where $E_{L}^{F}=1.35 . e V$ is the calculated formation energy in the lattice. We note that the binding energy can also be calculated by molecular dynamics techniques

TABLE I

Formation Energies, $E_{B}^{F}$, and Binding Energies, $E_{B}^{B}$, of Vacancies and Interstitials in Boundary Sites Shown in Fig. 1.

\begin{tabular}{cccc}
\hline & Boundary Site & $E_{B}^{F}(\mathrm{eV})$ & $E_{B}^{B}(e V)$ \\
\hline \multirow{2}{*}{ Vacancies } & A & 1.33 & -0.02 \\
& B & 0.94 & -0.41 \\
& C & 1.26 & -0.09 \\
& D & 1.17 & -0.18 \\
\hline Ir:tcrstitials & I & 1.06 & -3.68 \\
& A & 2.55 & -2.19 \\
& C & 2.55 & -2.19 \\
& D & 3.30 & -1.44 \\
& & 2.32 & -2.42 \\
\hline
\end{tabular}

using a damping force. Such a calculation has been carried out at site. B for the vacancy, and the result agrees with that calculated by the molecular statics method.

An interstitial was inserted by simply adding an extra atom in the boundary and again relaxing the structure. The largest "hole" in a hard sphere model of the perfect GB is located at the position I in Fig. 1, and an atom was therefore inserted at that point to simulate an interstitial. Considerable atomic relaxation occurred around this interstitial but it remained easily recognizable as an extra atom inserted at. I. Interstitials were also introduced at the sites $A$, $B, C$, and $D$ in Fig. 1. However, in these cases it was convenient to remove the atom originally at the site and then insert two atoms symmetrically disposed around the empty site. In the subsequent relaxation the two atoms were generally displaced, and the symetric arrangement was destroyed. These interstitials were therefore somewhat more delocalized but remained as bona fide point defects in the GB structure. The calculated formation and binding energies for these interstitials are also listed in Table 1 . (Here, the calculated formation energy in the lattice is $E_{L}^{F}=4.74 \mathrm{eV}$ for a $\langle 110\rangle$ split interstitial.)

\section{Molecular Dynamics Results}

The molecular dynamics of the GB model were investigated by adding thermal kinetic energy to the previously described static model. Standard molecular dynamics techniques (10,11) were used to simulate the behavior of the GB point defects as well as the atomic motions in the system. A number of isothermal "diffusion runs" was made in which a single vacancy was introduced in the $G B$ and observed to migrate as a function of time. The results are described in Ref. 5 and are briefly reviewed and discussed further below.

(i) The vacancy migrated in the core of the $G B$ by executing jumps between a variety of 


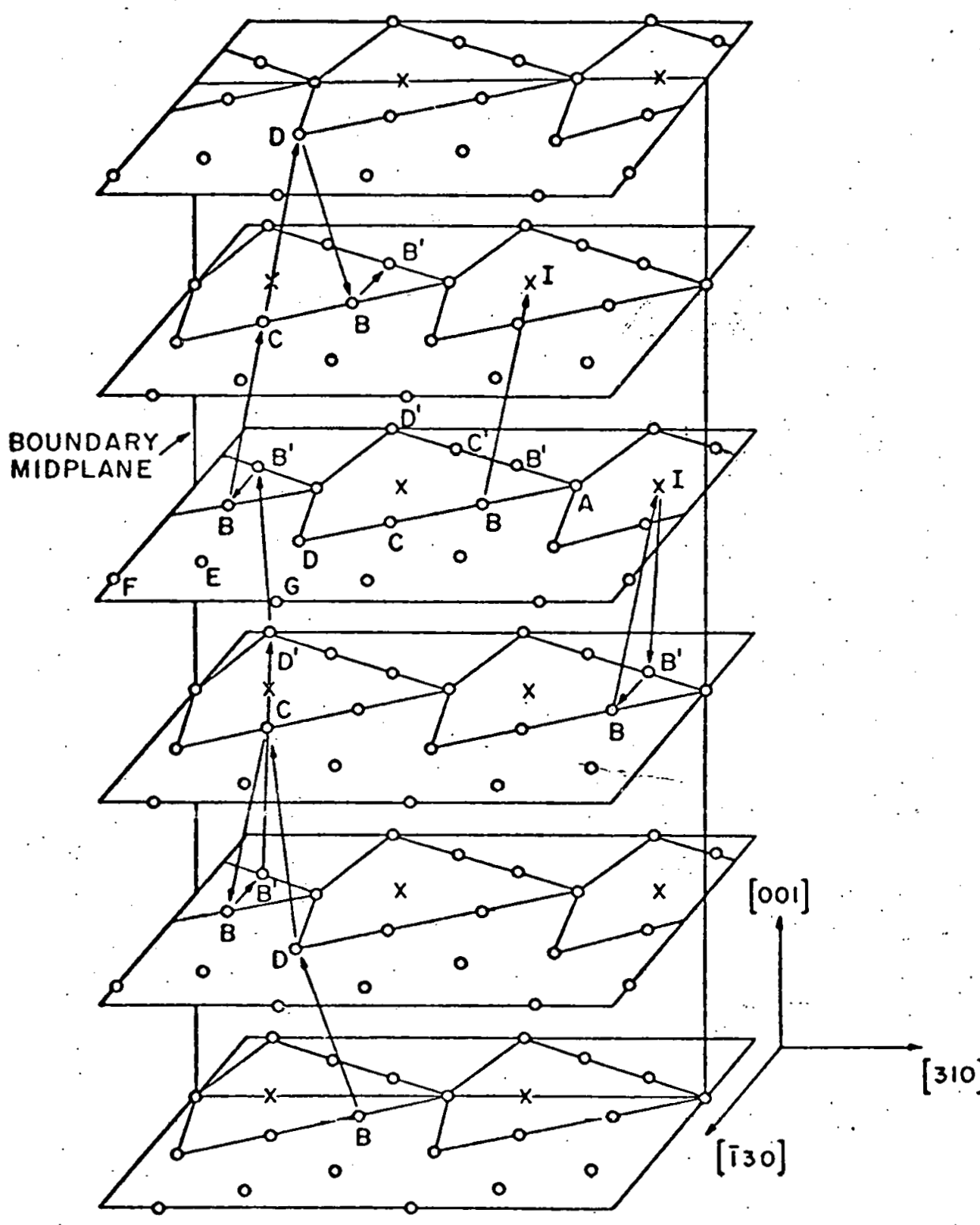

FIG. 1

Six of the 10 atomic planes used to model the bec iron [001] (310) tilt boundary $(\Sigma=5$, $\theta=36.87^{\circ}$ ) for computer simulation. Only half of the atoms in each plane (which contains four coincidence site lattice cells) are shown. A vacancy created at site $B$ preferentially junps among the boundary sites A, B, C, D rather than into the sites E, F, G which are further away from the boundary midplane. The sequence on the left indicates a typical vacancy jump path. The arrow in the conter shows an atom at $B$ jumping into the interstitial site $I$, thus creating a boundary interstitial and a boundary vacancy. The sequence on the right shows the observed interchange of atoms at $B$ and $B^{\prime}$ via a ring mechanism involving the interstitial site at $I$. The ratio of the scale uscd in the draving is $[130]:[310]:[001]=1: 1: 5$. 
TABLE II

Number of Vacancy Jumps into Various Boundary

Sites (Fig. 1) during Vacancy Diffusion Run Involving 195 Jumps at $1300 \mathrm{~K}$.

\begin{tabular}{l|ccccccc}
\hline Site & A & B & C & D & E & F & G \\
\hline $\begin{array}{c}\text { Number of } \\
\text { Jumps }\end{array}$ & 3 & 126 & 20 & 32 & 7 & 6 & 1 \\
\hline
\end{tabular}

sites. Essentially all jumps occurred between sites of the types labeled $A, B, C$, and $D$ in Fig.1. (Note that the $A^{\prime}, B^{\prime}, C^{\prime}$, and $D^{\prime}$ sites are equivalent sites because of the boundary symmetry.) The jumping was therefore confined almost entirely to the core of the GB. A typical trajectory is shown on the left of Fig. 1. The: number of times a vacancy jumped into a given type of site during a run at $1300 \mathrm{~K}$ involving 195 jumps is shown in Table II. Comparison of Table II with Table I shows a clear correlation between the vacancy binding energy at different sites and the. frequency with which a site received jumps; i.e., the larger the binding energy the more frequently it received jumps. The sites $E, F$, and $G$ are at distances from the GB midplane where the binding energy was relatively small, and they therefore received very few jumps.

(ii) The vacancy migration took place predominantly along the tilt axis rather than perpendicular to it (Fig. 1). sion:

(iii) The effective vacancy jump frequency (due to all jumps) obeyed the Arrhenius expres-

$$
\tilde{\Gamma}=\tilde{\Gamma}_{0} \exp \left(-\tilde{E}_{B}^{M} / k T\right)
$$

with $\tilde{\Gamma}_{0}=4.85 \times 10^{13} \mathrm{sec}^{-1}$, and $\tilde{\mathrm{E}}_{\mathrm{B}}^{\mathrm{M}}=0.51 \mathrm{ev}$. Here, $\tilde{\mathrm{E}}_{\mathrm{B}}^{\mathrm{M}}$ is the effective migration energy, and the pre-exponential factor can be written as $\tilde{\Gamma}_{0}=\tilde{z}_{\tilde{v}} \tilde{v}_{0}$, where $\tilde{z}=$ effective coordination number, and $\tilde{v}_{0}$ is an effective "attempt frequency". If $\tilde{z}=8, \tilde{v}_{0}=6.06 \times 10^{12} \mathrm{sec}^{-1}$, which is of the same magnitude as the Debye frequency (i.e., $7.1 \times 10^{12} \mathrm{sec}^{-1}$ ) as might be expected (12) if ef-

fects due to the entropy of migration are not large.

(iv) Atoms on B sites occasionally jumped into interstitial I sites by a process illustrated in the center of Fig. 1, which is essentially the thermally activated formation of a Frenkel pair. Inspection of Table I shows that this type of Frenkel pair must have a considerably lo:ver formation energy than any other possible Frenkel pair, and this is evidently the reason for its. occurrence. The vacancy formed in this way often diffused away leaving the interstitial behind at I. Furthermore, the interstitial at I remained completely immobile and could only be eliminated by mutual annihilation with a neighboring vacancy. The immobility of the interstitial at I is readily understood on the basis of the results in Table I where it is seen that the formation energies of the interstitial at the other interstitial sites are larger by at least $1.26 \mathrm{eV}$. The activation energy for the interstitial to migrate must therefore be larger than $1.26 \mathrm{eV}$, and this process was therefore not observed. These results indicate therefore that any interstitial in the GR will be strongly trapped at $I$ sites and will therefore be rendered immobile and incapable of promoting self-diffusion.

(v) The interchange of atoms at $B$ and $B^{\prime}$ by the process illustrated on the right side of Fig. I was observed occasionally. In this process an atom in site $B$ jumped into site $I$ in the adjacent boundary plane, followcd by an atom in site' $B^{\prime}$ jumping into the newly created vacancy in the site $B$. The process was then completed by the interstitial in the site I jumping into the vacancy sitting in site B'. This sequence was found to occur more frequently as the temperature increased. The process docs not contribute to diffusion because the two atoms involved remain trapped in the same pair configuration, thus producing no net matter transport relative to the other atoms.

\section{Discussion}

The previous results, taken all together, provide strong evidence for a vacancy exchange mechanism for self-diffusion in the present GB. We now show that we can derive an approximate 
expression for the $G B$ self-diffusion coefficient on the basis of a vacancy exchange model using the vacancy parameters that we have obtained. Furthermore, the expression predicts diffusivities which are in reasonable agreement with experimentally determined values. For a mechanism involving the exchange of atoms with vacancies in the GB core we may write (12) the self-diffusivity as :

$$
D_{B}=f \cdot g \cdot \tilde{\alpha}^{2} \cdot \tilde{N}_{v} \cdot \tilde{\Gamma},
$$

where $f=$ correlation factor, $g=$ geometrical factor, $\tilde{\alpha}^{2}=$ effective squared jump distance, and $\tilde{N}_{v}=$ effective equilibrium vacancy concentration. Therefore, by writing $\tilde{N}_{v}=\tilde{A}_{\text {exp }}\left(-\tilde{E}_{B}^{F} / k T\right)$, where $\tilde{A}=$ effective pre-exponential factor, and $\tilde{E}_{B}^{F}=$ effective formation energy, we have:

$$
D_{B} \simeq \tilde{D}_{B_{0}} \exp \left(-\tilde{Q}_{B} / k T\right)
$$

where

$$
\tilde{Q}_{B}=\tilde{E}_{B}^{F}+\tilde{E}_{B}^{M},
$$

and

$$
\tilde{D}_{B_{0}}=f \cdot g \cdot \tilde{\alpha}^{2} \cdot \tilde{A} \cdot \tilde{\Gamma}_{0}
$$

Consider first the quantity $\tilde{Q}_{B}$. Since the equilibrium vacancy population in the various GB sites in the core should obey a Boltzmann distribution we can take the concentration at each site as proportional to $\exp \left(-\tilde{E}_{B}^{F} / k T\right)$. If we assume that the constant of proportionality is the same for all sites we can estimate the total effective vacancy-concentration in the core (to within the constant of proportionality) by using the daca in Table I. The effective vacancy formation energy can then be found from the temperature dependence of the total concentration. The result of this procedure is $\tilde{E}_{B}^{F} \simeq 1.0 \mathrm{eV}$. Since the effective migration energy [see Eq. (1)] is $\tilde{E}_{B}^{M} \simeq 0.51 \mathrm{eV}$, we obtain $\tilde{Q}_{B} \simeq 1.51 \mathrm{eV}$.

Consider next the magnitude of the effective pre-exponential factor, $\tilde{D}_{B_{0}}$. The geometrical factor should be of order $g \simeq 1 / 3$. Since. there is a variety of vacancy jumps which occur in three dimensions in the boundary (i.e.., it does not consist of a single row of jump sites), we expect the correlation factor $f$ to be of order 0.5 (4). Also, reasonable values of $\tilde{A}$ and $\tilde{\alpha}^{2}$ are $\tilde{A}=\exp (2)$. (see Ref. 12), and $\tilde{\alpha}^{2}=\left[0.85 \mathrm{a}_{0}\right]^{2}$. Using these values and $\tilde{\Gamma}_{0}=4.85 \times 10^{13} \mathrm{sec}^{-1}$, we find $\ddot{D}_{B_{0}} \simeq 3.66 \times 10^{-2} \mathrm{~cm}^{2} \mathrm{sec}^{-1}$, and obtain finally:

$$
D_{B}=3.66 \times 10^{-2} \exp (-1.51 / \mathrm{kT})
$$

The value of $\tilde{Q}_{B}=1.51 \mathrm{eV}$ is significantly lower than the measured value of $2.5 \mathrm{eV}$ for lattice self-diffusion in bcc iron (13); and the pre-exponential factor, $3.66 \times 10^{-2} \mathrm{~cm}^{2} \mathrm{sec}^{-1}$, is in the range generally expected for this quantity. The calculated result therefore predicts rapid GB self-diffusion as required. Our conclusion about the relative magnitude of $\tilde{Q}_{B}$ agrees with one reáched cäriler by Brokmañ (14) on a more qualizarive basis.

Further examination shows that Eq. (6) predicts values of $D_{B}$ which are consistent with those reported in the literature. Martin and Perrailion (15) have recently plotted all available GB self-diffusion data on an Arrhenius plot using a reciprocal temperature scale normalized by the factor $1 / T_{m}$ where $T_{m}=$ melting temperature [see Fig. 1s(b) of Ref. 15]. The $D_{B}$ values given by Eq. (6) fall within the range of values appearing on the plot but they are generally lower than the average values by about an order of magnitude. In view of all the unccrtainties which are involved in the calculations we may thereforc regard the values of $D_{B}$ predicted by Eq. (6) as quite satisfactory. In this respect it is interesting to note that the present $G B$ is a 
relatively special boundary with a high coincidence site density misorientation and a short wave length periodicity (16). There is some evidence (4) that the GB self-diffusivity along such boundaries is generally slower than along more general boundaries. Since the data plotted in Fig. 18 (b) of Ref. 16 are representative of general boundaries, we may speculate that at least part of the above difference may be explained on this basis. We also note that the molecular dynamics simulation showed that the vacancy GB.migration was more rapid along the tilt axis than perpendicular to it (5). According to the vacancy exchange mechanism the GB self-diffusion rates should exhibit the same behavior, and this is confirmed experimentally (4).

In contrast to the behavior of vacancies in the GB core, interstitials appear to be relatively immobile and would therefore not be. expected to contribute significantly to GB selfdiffusion rates. The dynamics simulation (5) already demonstrated the immobility of the interstitial at the site $I$ (Fig. 1). Also, the results in Table I indicate that $\tilde{E}_{B}^{M}=1.26 \mathrm{eV}$ and $\tilde{E}_{B}^{F} \simeq 1.06$, and, therefore, $\tilde{Q}_{B} \simeq 2.32 \mathrm{eV}$. This result suggests that GB self-diffusion by an interstitial mechanism is probably slower than even lattice self-diffusion.

Finally, we mention that it would be interesting at this point to carry out further calculations of the present type for boundaries of different kinds. As has already been mentioned, the present $\sum=5$ tilt boundary is a rather special boundary in which the vacancies remain quite recognizable as empty sites. We have already shown $(8,9)$ that in other GBs the relaxation around vacancies may be greater causing them to dissociate or "split". The GB migration of such relaxed vacancies may be even easier than that of the present vacancies leading to even faster relative GB self-diffusion rates. Further calculations investigating this possibility are planned.

\section{Acknowledgment}

The U.S. Army Research Office provided support (for T.K. and S.Y.) under Contract DAAG-2978-C-0006. Support was provided by the U.S. Department of Energy (for R.H.B. and A.B.) and by the National Science Foundation (for R.W.B. and P.D.B.) under Contracts DE-AS02-78-ER0S002 and DMR 78-12804, respectively.

\section{References.}

1. F. B. Swinkels and M. F. Ashby, Acta Met. 29, 259 (1981).

2. B. Burton, Diffusional Creep of Polycrystalline Materials, Tech. Publ., Bay Vi.1lage, OH (1977).

3. R. W. Balluffi and J. W. Cahn, Acta Met. 29, 493 (1981).

4. N. L. Peterson, in: Grain Boundary Structure and Kinetics, American Society for Metals, Metals Park, OH (1980) p. 209.

5. Thomas Kwok, P. S. Ho, Sidney Yip, R. W. Balluffi, P. D. Bristowe and A. Brokman, to be published.

6. R. A. Johnson, Phys. Rev. 145, 423 (1966).

7. P. D. Bristowe and A. G. Crocker, Phil. Mag. 31, 503 (1975).

8. P. D. Bristowe, A. Brokman, F. Spaepen and R. W. Balluffi, Scripta Met. 14, 943 (1980).

9. A. Brokman, P. D. Bristowe and R. W. Balluffi, J. Appl. Phys., in press.

10. Interatonic Potentials and Simulation of Lattice Defects, eds., P. C. Gehlen, J. R. Beeler, R. I. Jaffee, Plenum Press, NY (1972).

11. G. H. Bishop, R. J. Harrison, T. Kwok and S. Yip, in: Progress in Materials Science, eds., B. Chalmers, J.W. Christian, T.B. Massalski, Pergamon Press, Oxford, in press.

12. P. G. Shewmon, Diffusion in Solids, McGraw-Hil1, NY (1963).

13. N. L. Peterson, J. Nucl. Mats. 69 and 70, 3 (1978).

14. A. Brokman, to be published.

15. G. Martin and B. Perraillon, in: Grain Boundary Structure and Kinetics, American Society for Metals, Metals Park, OH (1980) p. 239.

16. W. Bollmann, Crystal Defects and Crystalline Interfaces, Springer-Verlag, NY (1970) . 